\title{
A SYSTEM OF LINEAR RECURRENCE EQUATIONS FOR DETERMINANT OF PENTADIAGONAL MATRIX
}

\author{
Jolanta Borowska, Lena Lacińska, Jowita Rychlewska \\ Institute of Mathematics, Czestochowa University of Technology \\ Czestochowa, Poland \\ jolanta.borowska@im.pcz.pl,lena.lacinska@im.pcz.pl,jowita.rychlewska@im.pcz.pl
}

\begin{abstract}
In this paper we present an application of the system of two homogeneous linear recurrence equations to evaluate the determinant of pentadiagonal matrix. The general considerations are illustrated by two examples. It is shown that the proposed approach is suited for implementation using computer algebra systems.
\end{abstract}

Keywords: pentadiagonal matrix, determinant, linear recurrence equations

\section{Introduction}

The subject of consideration is a pentadiagonal matrix of the form

$$
A_{n}=\left[\begin{array}{ccccccccccc}
a_{1} & b_{1} & c_{1} & & & & & & & & \\
d_{2} & a_{2} & b_{2} & c_{2} & & & & & & & \\
e_{3} & d_{3} & a_{3} & b_{3} & c_{3} & & & & & & \\
& e_{4} & d_{4} & a_{4} & b_{4} & c_{4} & & & & & \\
& & \ddots & \ddots & \ddots & \ddots & \ddots & & & & \\
& & & \ddots & \ddots & \ddots & \ddots & \ddots & & & \\
& & & & e_{n-4} & d_{n-4} & a_{n-4} & b_{n-4} & c_{n-4} & & \\
& & & & & e_{n-3} & d_{n-3} & a_{n-3} & b_{n-3} & c_{n-3} & \\
& & & & & & e_{n-2} & d_{n-2} & a_{n-2} & b_{n-2} & c_{n-2} \\
& & & & & & & e_{n-1} & d_{n-1} & a_{n-1} & b_{n-1} \\
& & & & & & & & e_{n} & d_{n} & a_{n}
\end{array}\right]
$$

It means that $A_{n}=\left[a_{i j}\right]_{n \times n}$ where $a_{i j}=0$ for $|i-j|>2$. This type of matrices arises for example in a numerical solution of differential equations by using the finite element or finite difference methods. The aim of this paper is to derive a recurrence relation for determinant of matrix under considerations. The numerical algorithms for computing the determinant of pentadiagonal matrices were formulated in many 
papers, e.g. [1,2]. Most of these algorithms were obtained under certain assumptions. For example in [2] it was obtained that $\operatorname{det}\left(A_{n}\right)=\prod_{i=1}^{n} x_{i}$ if $x_{k} \neq 0$, $k=1,2, \ldots, n-1$ where

$$
\begin{gathered}
x_{i}=\left\{\begin{array}{ll}
a_{1} & i=1 \\
a_{2}-y_{1} z_{2} & i=2 \\
a_{i}-y_{i-1} z_{i}-\frac{e_{i}}{x_{i-2}} c_{i-2} & i=3, \ldots, n
\end{array} \quad, y_{i}= \begin{cases}b_{1} & i=1 \\
b_{i}-z_{i} c_{i-1} & i=2, \ldots, n-1\end{cases} \right. \\
z_{i}= \begin{cases}\frac{d_{2}}{x_{1}} \\
\frac{d_{i}-\frac{e_{i}}{x_{i-2}} y_{i-2}}{x_{i-1}} & i=3, \ldots, n\end{cases}
\end{gathered}
$$

In the present paper we are to show that determinant of pentadiagonal matrix of the form (1) is a particular solution of a system of two linear recurrence equations. This approach is available for every pentadiagonal matrix.

\section{The main results}

In order to derive recurrence relation for determinant of matrix (1) we introduce two auxiliary pentadiagonal matrices of the form

$$
\widetilde{A}_{n}=\left[\begin{array}{ccccccccccc}
a_{1} & b_{1} & c_{1} & & & & & & & & \\
d_{2} & a_{2} & b_{2} & c_{2} & & & & & & & \\
e_{3} & d_{3} & a_{3} & b_{3} & c_{3} & & & & & & \\
& e_{4} & d_{4} & a_{4} & b_{4} & c_{4} & & & & & \\
& & \ddots & \ddots & \ddots & \ddots & \ddots & & & & \\
& & & \ddots & \ddots & \ddots & \ddots & \ddots & & & \\
& & & & e_{n-4} & d_{n-4} & a_{n-4} & b_{n-4} & c_{n-4} & & \\
& & & & & e_{n-3} & d_{n-3} & a_{n-3} & b_{n-3} & c_{n-3} & \\
& & & & & & e_{n-2} & d_{n-2} & a_{n-2} & b_{n-2} & 0 \\
& & & & & & & e_{n-1} & d_{n-1} & a_{n-1} & c_{n-1} \\
& & & & & & & & e_{n} & d_{n} & b_{n}
\end{array}\right]
$$


and

$$
\bar{A}_{n}=\left[\begin{array}{cccccccccccc}
a_{1} & b_{1} & c_{1} & & & & & & & & \\
d_{2} & a_{2} & b_{2} & c_{2} & & & & & & & \\
e_{3} & d_{3} & a_{3} & b_{3} & c_{3} & & & & & & \\
& e_{4} & d_{4} & a_{4} & b_{4} & c_{4} & & & & & \\
& & \ddots & \ddots & \ddots & \ddots & \ddots & & & & \\
& & & \ddots & \ddots & \ddots & \ddots & \ddots & & & \\
& & & & e_{n-4} & d_{n-4} & a_{n-4} & b_{n-4} & c_{n-4} & & \\
& & & & & e_{n-3} & d_{n-3} & a_{n-3} & b_{n-3} & 0 & \\
& & & & & & e_{n-2} & d_{n-2} & a_{n-2} & c_{n-2} & 0 \\
& & & & & & & e_{n-1} & d_{n-1} & b_{n-1} & c_{n-1} \\
& & & & & & & & e_{n} & a_{n} & b_{n}
\end{array}\right]
$$

Let us denote

$$
W_{n}=\operatorname{det} A_{n}, \widetilde{W}_{n}=\operatorname{det} \widetilde{A}_{n}, \bar{W}_{n}=\operatorname{det} \bar{A}_{n}
$$

Using the method of Laplace expansion with respect to the last row of matrix $A_{n}$ we obtain

$$
W_{n}=a_{n} W_{n-1}-d_{n} \tilde{W}_{n-1}+e_{n} \bar{W}_{n-1}
$$

Now, we are to derive recurrence relation for determinant $\widetilde{W}_{n-1}$. To this end we use Laplace expansion with respect to last column of matrix $\widetilde{A}_{n-1}$, which leads to the linear combination of two determinants. First of these determinants is equal to $W_{n-2}$ and for second determinant we apply Laplace expansion with respect to the last row and we obtain a linear combination of determinants $W_{n-3}$ and $\tilde{W}_{n-3}$. Hence

$$
\tilde{W}_{n-1}=b_{n-1} W_{n-2}-c_{n-2}\left(d_{n-1} W_{n-3}-e_{n-1} \tilde{W}_{n-3}\right)
$$

Subsequent considerations will be concerned with determinant $\bar{W}_{n-1}$. We start with Laplace expansion with respect to the last column of matrix $\bar{A}_{n-1}$, which leads to the linear combination of two determinants. The first of these determinants is equal to $\widetilde{W}_{n-2}$ and for the second determinant we apply Laplace expansion with respect to the last row and we obtain a linear combination of two determinants. The first of them is equal to $W_{n-3}$ and for the second determinant we use Laplace expansion 
with respect to the last column, which leads to the linear combination of determinants $W_{n-3}$ and $W_{n-4}$. Finally we get

$$
\bar{W}_{n-1}=b_{n-1} \widetilde{W}_{n-2}-c_{n-2}\left(a_{n-1} W_{n-3}-e_{n-1} c_{n-3} W_{n-4}\right)
$$

Bearing in mind relations (2), (3) and (4) we obtain a system of two linear recurrence equations

$$
\left\{\begin{array}{l}
W_{n}=a_{n} W_{n-1}-e_{n} c_{n-2} a_{n-1} W_{n-3}+c_{n-2} c_{n-3} e_{n-1} e_{n} W_{n-4}-d_{n} \widetilde{W}_{n-1}+e_{n} b_{n-1} \widetilde{W}_{n-2} \\
\widetilde{W}_{n-2}=b_{n-2} W_{n-3}-c_{n-3} d_{n-2} W_{n-4}+c_{n-3} e_{n-2} \widetilde{W}_{n-4}
\end{array}\right.
$$

where $n>4$.

The above equations can be rewritten in the following form

$$
\left\{\begin{array}{l}
W_{n+4}=a_{n+4} W_{n+3}-e_{n+4} c_{n+2} a_{n+3} W_{n+1}+c_{n+2} c_{n+1} e_{n+3} e_{n+4} W_{n}-d_{n+4} \widetilde{W}_{n+3}+e_{n+4} b_{n+3} \widetilde{W}_{n+2} \\
\widetilde{W}_{n+2}=b_{n+2} W_{n+1}-c_{n+1} d_{n+2} W_{n}+c_{n+1} e_{n+2} \widetilde{W}_{n}
\end{array}\right.
$$

where $n \in \mathbf{N}$.

In order to obtain determinant $W_{n}$ of matrix $A_{n}$ we must take into account the system of equations (6) together with the initial conditions of the form

$$
\left\{\begin{array}{l}
\widetilde{W}_{1}=b_{1} \\
\widetilde{W}_{2}=a_{1} b_{2}-c_{1} d_{2} \\
W_{1}=a_{1} \\
W_{2}=a_{1} a_{2}-b_{1} d_{2} \\
W_{3}=a_{3} W_{2}-d_{3} \widetilde{W}_{2}+e_{3}\left(b_{1} b_{2}-c_{1} a_{2}\right) \\
W_{4}=a_{4} W_{3}-d_{3} \widetilde{W}_{3}+e_{4} b_{3} \widetilde{W}_{2}+e_{4} c_{2}\left(c_{1} e_{3}-a_{1} a_{3}\right)
\end{array}\right.
$$

Hence the value of determinant of pentadiagonal matrix $A_{n}$ is the particular solution of the system of equations (6) fulfilling initial conditions (7). It can be observed that the direct solution of the system of equations (6) can be obtained only in some special cases. However, for an arbitrary but fixed $n \in \mathbf{N}$ we can find determinant $W_{n}$ using computer algebra systems such as Maple, Mathematica and Matlab.

\section{Remark 1.}

If $\left(a_{k}\right)_{k=1}^{n}=a,\left(b_{k}\right)_{k=1}^{n-1}=0,\left(c_{k}\right)_{k=1}^{n-2}=c,\left(d_{k}\right)_{k=2}^{n}=0,\left(e_{k}\right)_{k=3}^{n}=e$ in matrix (1), then we have 


$$
A_{n}=\left[\begin{array}{ccccccc}
a & 0 & c & & & & \\
0 & a & 0 & c & & & \\
e & 0 & a & 0 & c & & \\
& \ddots & \ddots & \ddots & \ddots & \ddots & \\
& & \ddots & \ddots & \ddots & \ddots & c \\
& & & e & 0 & a & 0 \\
& & & & e & 0 & a
\end{array}\right]_{n \times n}
$$

In this case the system of equations (6) reduces to one recurrence equation of the form

$$
W_{n+4}=a W_{n+3}-a c e W_{n+1}+e^{2} c^{2} W_{n}
$$

with initial conditions

$$
W_{1}=a, W_{2}=a^{2}, W_{3}=a^{3}-a c e, W_{4}=a^{4}-2 a^{2} c e+e^{2} c^{2}
$$

Hence the determinant of matrix (8) is the particular solution of equation (9) fulfilling initial conditions (10). The above result was presented in paper [3].

\section{Remark 2.}

If $\left(c_{k}\right)_{k=1}^{n-2}=0,\left(e_{k}\right)_{k=3}^{n}=0$ in (1), then we obtain the tridiagonal matrix of the form

$$
A_{n}=\left[\begin{array}{ccccccc}
a_{1} & b_{1} & & & & & \\
d_{2} & a_{2} & b_{2} & & & & \\
& d_{3} & a_{3} & b_{3} & & & \\
& & d_{4} & a_{4} & b_{4} & & \\
& & & \ddots & \ddots & \ddots & \\
& & & & d_{n-1} & a_{n-1} & b_{n-1} \\
& & & & & d_{n} & a_{n}
\end{array}\right]_{n \times n}
$$

In this case system of equations (6) reduces to one recurrence equation of the form

$$
W_{n+2}=a_{n+2} W_{n+1}-d_{n+2} b_{n+1} W_{n}
$$

fulfilling initial conditions

$$
W_{1}=a_{1}, W_{2}=a_{1} a_{2}-b_{1} d_{2}
$$

Hence the determinant of tridiagonal matrix (11) is the particular solution of equation (12) fulfilling initial conditions (13). 


\section{Illustrative examples}

Now, we are to illustrate the general results obtained in the previous section.

\section{Example 1.}

Now, we consider matrix (1) of order $n \times n$ setting $\left(a_{k}\right)_{k=1}^{n}=1,\left(b_{k}\right)_{k=1}^{n-1}=0$, $\left(c_{k}\right)_{k=1}^{n-2}=\frac{1}{k},\left(d_{k}\right)_{k=2}^{n}=0,\left(e_{k}\right)_{k=3}^{n}=k-2$. From (6) the determinant of this matrix is given by the formula

$$
W_{n+4}-W_{n+3}+W_{n+1}-W_{n}=0
$$

with initial conditions

$$
W_{1}=1, W_{2}=\left|\begin{array}{ll}
1 & 0 \\
0 & 1
\end{array}\right|=1, W_{3}=\left|\begin{array}{ccc}
1 & 0 & 1 \\
0 & 1 & 0 \\
1 & 0 & 1
\end{array}\right|=0, W_{4}=\left|\begin{array}{cccc}
1 & 0 & 1 & 0 \\
0 & 1 & 0 & 1 / 2 \\
1 & 0 & 1 & 0 \\
0 & 2 & 0 & 1
\end{array}\right|=0
$$

Equation (14) is a fourth-order homogeneous linear recurrence equation with constant coefficients. Following [4] we have that the general solution of equation (14) is determined by the roots of the characteristic equation

$$
\lambda^{4}-\lambda^{3}+\lambda-1=0
$$

Roots of (16) are equal to $\lambda_{1}=1, \lambda_{2}=-1, \lambda_{3}=\frac{1}{2}-\frac{\sqrt{3}}{2} i, \lambda_{4}=\frac{1}{2}+\frac{\sqrt{3}}{2} i$. Hence the general solution of equation (14) has the form

$$
W_{n}=C_{1}+C_{2}(-1)^{n}+C_{3} \cos \frac{n \pi}{3}+C_{4} \sin \frac{n \pi}{3}
$$

Taking into account initial conditions (15) we obtain the system of linear equations

$$
\left[\begin{array}{rrcc}
1 & -1 & 1 / 2 & \sqrt{3} / 2 \\
1 & 1 & -1 / 2 & \sqrt{3} / 2 \\
1 & -1 & -1 & 0 \\
1 & 1 & -1 / 2 & -\sqrt{3} / 2
\end{array}\right]\left[\begin{array}{l}
C_{1} \\
C_{2} \\
C_{3} \\
C_{4}
\end{array}\right]=\left[\begin{array}{l}
1 \\
1 \\
0 \\
0
\end{array}\right]
$$

Hence

$$
C_{1}=\frac{1}{2}, C_{2}=\frac{1}{6}, C_{3}=\frac{1}{3}, C_{4}=\frac{\sqrt{3}}{3}
$$


Substituting (18) to (17) we have the particular solution of equation (14) with initial conditions (15) in the form

$$
W_{n}=\frac{1}{2}+\frac{1}{6}(-1)^{n}+\frac{1}{3} \cos \frac{n \pi}{3}+\frac{\sqrt{3}}{3} \sin \frac{n \pi}{3}
$$

Formula (19) represents the determinant of the matrix under consideration.

\section{Example 2.}

Let us consider a special form of pentadiagonal matrix (1) in which elements on diagonals are defined by sequences of the form $\left(a_{k}\right)_{k=1}^{n}=k^{2},\left(b_{k}\right)_{k=1}^{n-1}=k+1$, $\left(c_{k}\right)_{k=1}^{n-2}=2 k-3,\left(d_{k}\right)_{k=2}^{n}=3 k+2,\left(e_{k}\right)_{k=3}^{n}=2 k^{2}$. Moreover, assume that $n=10^{4}$, i.e. matrix has the order $10^{4} \times 10^{4}$. Bearing in mind (6) the determinant of this matrix is given by the system of two linear recurrence equations with functional coefficients of the form

$$
\left\{\begin{aligned}
W_{n+4} & =(n+4)^{2} W_{n+3}-2(n+4)^{2}(2 n+1)(n+3)^{2} W_{n+1}+ \\
& +4(2 n+1)(2 n-1)(n+3)^{2}(n+4)^{2} W_{n}-(3 n+5) \widetilde{W}_{n+3}+2(n+4)^{2}(n+4) \widetilde{W}_{n+2} \\
\widetilde{W}_{n+2} & =(n+3) W_{n+1}-(2 n-1)(3 n+5) W_{n}+2(2 n-1)(n+2)^{2} \widetilde{W}_{n}, \quad n=1,2, \ldots, 10^{4}-4
\end{aligned}\right.
$$

with initial conditions

$$
\widetilde{W}_{1}=2, \quad \widetilde{W}_{2}=11, \quad W_{1}=1, \quad W_{2}=-12, \quad W_{3}=-49, \quad W_{4}=82
$$

Let us observe that now we are dealing with a system of two linear recurrence equations with functional coefficients. It is impossible to solve this system using known analytical methods. Therefore, we use the Maple system in order to calculate the determinant of the matrix under consideration. To this end let us denote $F=\widetilde{W}$ and apply the following syntax

$a:=\left[\operatorname{seq}\left(n^{2}, n=1 . .10000\right), 0\right]:$

$b:=[\operatorname{seq}(n+1, n=1 . .10000), 0]:$

$c:=[\operatorname{seq}(2 n-3, n=1 . .10000), 0]:$

$d:=[0, \operatorname{seq}(3 n+2, n=2 . .10000), 0]:$

$e:=\left[0,0, \operatorname{seq}\left(2 n^{2}, n=3 . .10000\right), 0\right]:$

$F[1]:=b[1]:$

$F[2]:=a[1] \cdot b[2]-c[1] \cdot d[2]:$

$W[1]:=1$ : 
$W[2]:=-12:$

$W[3]:=-49$ :

$W[4]:=82$ :

for $n$ from 1 to 9997 do

$W[n+4]:=a[n+4] \cdot W[n+3]-e[n+4] \cdot c[n+2] \cdot a[n+3] \cdot W[n+1]+$ $c[n+2] \cdot c[n+1] \cdot e[n+3] \cdot e[n+4] \cdot W[n]-d[n+4] \cdot F[n+3]+$ $e[n+4] \cdot b[n+3] \cdot F[n+2]:$

$F[n+2]:=c[n+1] \cdot e[n+2] \cdot F[n]+b[n+2] \cdot W[n+1]-c[n+1] \cdot d[n+2] \cdot W[n]:$ end do:

$\operatorname{print}(\operatorname{evalf}(W[10000]))$

Finally we get $1.414983547 \times 10^{71299}$ as the value of the determinant of the matrix under consideration. It can be emphasized that the above result was obtained with Maple default precision (Digits $=10)$.

\section{Conclusions}

It was shown that the determinant of the pentadiagonal matrix can be obtained as particular solution of the system of two homogeneous linear recurrence equations. The general considerations was illustrated by two examples. In Example 1 the direct formula for determinant was obtained. In Example 2 the implementation of the proposed approach to Maple was presented. Moreover, it was presented that the above way leads to one linear recurrence equation for a determinant of the tridiagonal matrix.

\section{References}

[1] Aiat Hadj A.D., Elouafi M., On the characteristic polynomial, eigenvectors and determinant of a pentadiagonal matrix, Applied Mathematics and Computation 2008, 198, 634-642.

[2] Zhao X., Huang T., On the inverse of a general pentadiagonal matrix, Applied Mathematics and Computation 2008, 202, 639-646.

[3] Borowska J., Łacińska L., Rychlewska J., On determinant of certain pentadiagonal matrix, Journal of Applied Mathematics and Computational Mechanics 2013, 12(3), 21-26.

[4] Elaydi S., An Introduction to Difference Equations, Springer, 2005. 